\title{
Deep learning-based quantification of temporalis muscle has prognostic value in patients with glioblastoma
}

\author{
Ella Mi ${ }^{1,2,6}$, Radvile Mauricaite ${ }^{1,2,6}$, Lillie Pakzad-Shahabi ${ }^{1,3}$, Jiarong Chen ${ }^{1,4}$, Andrew Ho ${ }^{1,5}$ and Matt Williams $\mathbb{D}^{1,2}$ \\ (c) The Author(s) 2021
}

BACKGROUND: Glioblastoma is the commonest malignant brain tumour. Sarcopenia is associated with worse cancer survival, but manually quantifying muscle on imaging is time-consuming. We present a deep learning-based system for quantification of temporalis muscle, a surrogate for skeletal muscle mass, and assess its prognostic value in glioblastoma.

METHODS: A neural network for temporalis segmentation was trained with 366 MRI head images from 132 patients from 4 different glioblastoma data sets and used to quantify muscle cross-sectional area (CSA). Association between temporalis CSA and survival was determined in 96 glioblastoma patients from internal and external data sets.

RESULTS: The model achieved high segmentation accuracy (Dice coefficient 0.893). Median age was 55 and 58 years and 75.6 and $64.7 \%$ were males in the in-house and TCGA-GBM data sets, respectively. CSA was an independently significant predictor for survival in both the in-house and TCGA-GBM data sets (HR 0.464,95\% Cl 0.218-0.988, $p=0.046 ; \mathrm{HR} 0.466,95 \% \mathrm{Cl} 0.235-0.925, p=$ 0.029 , respectively).

CONCLUSIONS: Temporalis CSA is a prognostic marker in patients with glioblastoma, rapidly and accurately assessable with deep learning. We are the first to show that a head/neck muscle-derived sarcopenia metric generated using deep learning is associated with oncological outcomes and one of the first to show deep learning-based muscle quantification has prognostic value in cancer.

British Journal of Cancer (2022) 126:196-203; https://doi.org/10.1038/s41416-021-01590-9

\section{INTRODUCTION}

Glioblastoma multiforme (GBM) is an aggressive brain malignancy with $<5 \% 5$-year survival [1]. Factors including age, performance status, tumour location, size, molecular and histological characteristics are known to be prognostic, with performance status particularly important [2]. However, performance status is subjectively evaluated, resulting in inaccuracy and high interobserver variability, so objectively assessable indicators of frailty/ physical condition such as measures of sarcopenia and skeletal muscle mass may improve prognostic assessment and treatment stratification.

Sarcopenia is associated with worse overall (OS) and progression-free survival (PFS), postoperative outcomes and chemotherapy toxicity in common cancer types [3-8]. It can be evaluated on cross-sectional imaging that is routinely performed on cancer patients. Methods with consensus include measuring cross-sectional area (CSA) of truncal abdominal musculature at L3 and psoas muscle on computed tomography (CT); these have been associated with survival in numerous cancers $[4,6,8]$. However, GBM patients routinely undergo magnetic resonance imaging (MRI) head during follow-up, rather than body $C T$, so there is a need for measures of sarcopenia and skeletal muscle mass derivable from MRI head. The most widely studied is temporalis muscle width (TMW), which has been identified as a skeletal muscle mass surrogate [9] and a prognostic factor for OS and PFS in GBM [10-13] and brain metastases [14, 15]. However, no studies have investigated temporalis CSA, which is likely to be a better indicator of muscle mass than width.

Currently, assessment of muscle dimensions on cancer imaging is by manual measurement or segmentation, which is time consuming, necessitates trained personnel and is prone to interrater inconsistency, thus limiting application to large data sets and clinical adoption. Automated muscle segmentation techniques are required for widespread use.

Automated methods have been developed for muscle segmentation, including thresholding, fuzzy c-means clustering, atlas/ registration-based methods and shape prior modelling. One study applied range-constrained thresholding and adaptive morphological operations to segment temporalis [16], while another used Markov random field approach and region growing [17]. However, these have shortcomings: thresholding may fail when neighbouring tissues have similar intensity (as with facial muscles), atlas/ registration-based methods require high computational resources and substantial time to segment each case and can fail to locate

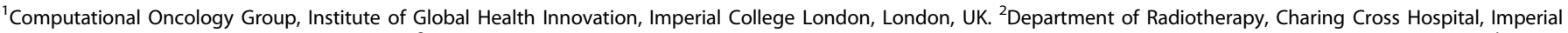

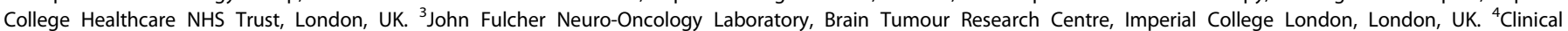

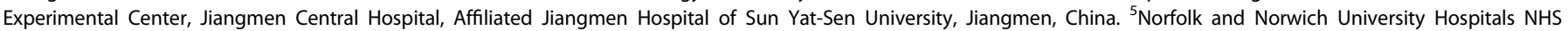
Foundation Trust, Norwich, UK. ${ }^{6}$ These authors contributed equally: Ella Mi, Radvile Mauricaite. ${ }^{凶}$ email: matthew.williams@imperial.ac.uk 
complex facial muscle structures with sufficient precision. Additionally, these methods are often semi-automatic, requiring prior knowledge, complicated feature selection and error correction, which make them challenging to build into clinical workflows.

In recent years, deep learning methods, in particular convolutional neural networks (CNNs), have achieved great success in medical image segmentation. A number of studies have demonstrated utility of CNNs in thigh [18, 19], abdominal [20-24], pelvic [25] and shoulder muscle segmentation [26, 27] for body composition analysis and sarcopenia assessment in populationbased cohorts and disease conditions. However, work applying deep learning to muscle segmentation in cancer imaging or muscle segmentation on head scans is limited. In particular, no studies have provided or evaluated the use of a scalable, deep learning-based approach to quantify the temporalis muscle or its relationship to survival in brain tumour patients.

The aim of this study was to develop and validate a deep learning system for segmentation and quantification of temporalis muscle and determine whether muscle area predicts disease progression and survival in GBM.

\section{METHODS}

Data

For training, validation and testing of segmentation performance, we used three-dimensional (3D) MRI head scans from four different data sets: an inhouse glioblastoma data set (a retrospective cohort of patients with newly diagnosed GBM presenting between January 2015 and May 2018 to a tertiary medical centre) $(n=40)$, TCGA-GBM $(n=31)$, IVY-GAP $(n=23)$, and REMBRANDT $(n=38)$ (the latter three from The Cancer Imaging Archive [28]). The methods of this study have been described in part in Mauricaite et al. [29].

For the survival analysis, MRI head scans were obtained from two data sets with corresponding clinical data for patients-our in-house glioblastoma patient data set $(n=45)$ and TCGA-GBM $(n=51)$. Inclusion criteria were patients with histologically proven GBM and available baseline preoperative MRI head. The total number of glioblastoma patients in the in-house data set was 45 but we only used some of these scans as access to some was obtained after model training.

All scans were T1-weighted MRI sequences acquired with gadolinium contrast in the axial plane.

For patients in the in-house glioblastoma data set, patients' age, sex, MGMT promoter methylation status and dates of diagnosis, death and progression were collected by LP-S who was blinded to model development and evaluation and quantification of temporalis CSA on patient scans. OS and PFS were calculated as time from date of diagnosis to death and progression, respectively. Both were censored at the date of last follow-up. For patients in the TCGA-GBM data set, clinical data were obtained from the TCGA repository. Dates of disease progression were not available.

In-house data were used in accordance with REC/HRA approval (reference: 19/LO/1763 IRAS ID: 265404) and conducted in accordance with this committee's regulations and the Declaration of Helsinki. Public data (TCGA-GBM, IVY-GAP, REMBRANDT) was used in accordance with permissions for those data sets.

\section{In-house MRI scanning}

All in-house scans were acquired on a $3 \mathrm{~T}$ Siemens scanner (Siemens Healthineers, Erlangen, Germany), with administration of intravenous gadolinium contrast. Protocols used were: two-dimensional (2D) fast low angle shot (FLASH), 3D magnetisation prepared rapid gradient echo (MPRAGE) and spin echo sequence, with field of view $=158-260 \mathrm{~mm} \times$ $218-260 \mathrm{~mm}$, matrix $=176-512 \times 256-512$ and slice thickness $=1-5 \mathrm{~mm}$.

\section{Image preprocessing}

MRIcroGL [30] was used to convert images from DICOM to NIfTI. Advanced Normalisation tools for Python [31] and the Intensity-Normalisation package [32] were used for bias-field correction and Z-score normalisation. Resampling of images to $1 \times 1$ voxel spacing and resizing to $256 \times 256$ pixels was performed.

\section{Image extraction}

MRI head scans were sliced into 2D image sequences. For training and testing of the neural network, $3662 \mathrm{D}$ axial MRI slices from 132 patient scans were extracted at levels on the craniocaudal axis between the midorbit and orbital roof.

\section{Manual segmentation}

The temporalis muscle was manually segmented on the MRI slices, creating a binary mask of pixels assigned to either muscle or background class. Segmentations were performed using ITK-SNAP v3.2 [33] by consensus of two specially trained readers, RM and EM, an oncology specialty doctor, who received instruction from a senior neuro-radiologist AG with 11 years of experience. Segmentation maps were independently reviewed by MW, a senior neuro-oncologist with 9 years of experience, and $\mathrm{JC}$, a neuro-oncologist with 5 years of experience. These served as reference ground truth labels in model training and evaluation. All readers were blinded to demographic and clinical characteristics of patients.

\section{Training, validation and test data sets}

The 366 2D MR slices with corresponding ground truth labels from 132 patients were split into training, validation and test sets: 229 slices (74 patients) for training, 65 slices ( 27 patients) for validation, and 72 slices (31 patients) were held out for use in a test set to evaluate model performance. For patients in the validation and test sets, three slices were taken from their scan if it was from the in-house glioblastoma patient data set and two slices were taken from their scan if it was from any of the external data sets (as the in-house data set scans had significantly more total slices than scans from the external data sets). Hence, in the validation and test sets, the minimum number of slices per scan was two and the maximum was three. For patients in the training set, 3-10 slices were taken from their scan if it was from the in-house glioblastoma patient data set and 2 slices were taken from their scan if it was from any of the external data sets. More slices were taken from scans in the in-house glioblastoma patient data set as they had a larger number of total slices (some had $>500$ slices), in order to increase the size of the training set.

\section{CNN model}

A deep neural network was trained for temporalis muscle segmentation, based on Ronneberger et al.'s 2D U-net architecture [34, 35], which takes MR images and ground truth muscle segmentations and yields predicted muscle segmentations. U-net is a CNN, with symmetric contracting and expanding paths. The contracting path consists of series of two $3 \times 3$ convolutional layers and one $2 \times 2$ max pooling layer, which downsample and convert input images into high-dimensional feature maps, enabling extraction of salient image features. Conversely, the expanding path consists of $2 \times 2$ deconvolutional and $3 \times 3$ convolutional layers, which upsample and retrieve image resolution from feature maps, enabling exact pixel-level localisation. The expanding path is followed by a $1 \times 1$ convolutional layer with sigmoid activation, which outputs the probability of each pixel being classed as muscle; this is binarised at 0.5 threshold such that each pixel is allocated to either muscle or background class. The deep neural network we constructed used 4 downsampling and upsampling operations and the same padding to convolutional kernels, reducing a $256 \times 256$ pixel image to a $16 \times 16$ data representation with 1024 channels. Regularisation was added with batch normalisation after each convolutional layer and two dropout layers (with dropout rate of 0.5 ) in order to reduce overfitting.

\section{CNN training}

The U-Net was trained using stochastic gradient descent with Adam optimiser on a mini batch size of two. Optimal hyperparameters were selected based on validation loss. Initial learning rates of $0.0001,0.0005$, $0.00075,0.001$ and 0.01 were tested. Training was terminated early if the validation loss did not improve in three subsequent epochs. The best performing model was trained for 14 epochs. We implemented our model using the Tensorflow v1.14.0 [36] and Keras v2.4 [37] libraries in Python v3.6 [38]. Experiments were run on a NVIDIA GeForce RTX 2080Ti GPU.

\section{Loss functions}

We previously compared three main categories of loss function-binary cross-entropy loss (BCEL) (distribution-based), Dice loss (DL) (regionbased), and Hausdorff loss (HDL) (boundary-based)—in training and 
Table 1. Segmentation metrics for U-Net models trained with Dice, binary cross-entropy and Hausdorff loss functions on the in-house glioblastoma MRI data set.

\begin{tabular}{llllll} 
Metric & Dice loss & Binary cross-entropy loss & DL vs BCEL $\boldsymbol{p}$ value & Hausdorff loss & DL vs HDL $\boldsymbol{p}$ value \\
\hline DSC & $0.9124 \pm 0.0310$ & $0.8931 \pm 0.0397$ & $<0.0005^{*}$ & $0.7938 \pm 0.1326$ & $<0.0005^{*}$ \\
\hline JI & $0.8404 \pm 0.0514$ & $0.8091 \pm 0.0623$ & $<0.0005^{*}$ & $0.6730 \pm 0.1421$ & $<0.0005^{*}$ \\
\hline Precision & $0.9253 \pm 0.0557$ & $0.9164 \pm 0.0714$ & 0.155 & $0.8571 \pm 0.0599$ & $<0.0005^{*}$ \\
Recall & $0.9033 \pm 0.0448$ & $0.8761 \pm 0.0494$ & $0.002^{*}$ & $0.7660 \pm 0.1751$ & $<0.0005^{*}$ \\
\hline HD $(\mathrm{mm})$ & $1.8129 \pm 0.3895$ & $1.8311 \pm 0.3433$ & 0.793 & $2.1787 \pm 0.4393$ & $<0.0005^{*}$ \\
\hline
\end{tabular}

All figures are mean \pm SD.

DSC Dice coefficient, J Jaccard index, HD Hausdorff distance, DL Dice loss, BCEL binary cross-entropy loss, HDL Hausdorff loss.

*Significant at $p<0.05$.

testing of the U-net model using the in-house glioblastoma data set only. BCEL and DL can be represented:

Binary cross-entropy loss $=-\frac{1}{N} \sum_{i=1}^{N} g i \cdot \log (p i)+(1-g i) \cdot \log (1-p i)$

Dice loss $=1-\frac{2 \sum_{i}^{N} p i \cdot g i}{\sum_{i}^{N} p i+\sum_{i}^{N} g i}$

where $N$ is the total number of image pixels and $g i$ and $p i$ are the $i$ th pixel of the ground truth and predicted segmentations, respectively. We used the DL implementation of an open-source image segmentation library [39] and $\mathrm{HDL}$ as derived in Ribera et al. $[40,41]$.

\section{Model evaluation}

Following training, the model was evaluated on accuracy and generalisability on the test set of unseen MR images. Comparison between ground truth and predicted segmentations were made on metrics of Dice similarity coefficient (DSC), Jaccard index (Jl), precision, recall and Hausdorff distance (HD) (Supplement A).

CSA of the muscle segmentation was computed using OpenCV [42]; from this, we calculated an additional evaluation metric of CSA error (Supplement B).

\section{Survival analysis}

We analysed the association between temporalis CSA and survival in 96 patients, 45 from our in-house glioblastoma data set and 51 from the external TCGA-GBM data set. For the in-house data set, to determine CSA, a single MRI slice was taken from each of the 45 scans at the level of the superior orbital quarter, defined as equidistant between the orbital roof and mid-orbit, the level of maximal temporalis CSA. For the TCGA-GBM data set, a single MRI slice was taken from each of the 51 scans at the level of the mid-orbit.

The trained CNN model was used to generate predicted segmentations for both the left and right temporalis muscles on each MRI slice and temporalis muscle CSAs were computed from these segmentations. Average CSA for each patient was calculated as the mean of the CSAs of the two temporalis muscles.

Median temporalis CSA was determined separately for the in-house and TCGA cohort and used to dichotomise patients in each cohort to 'low'/ 'high' CSA groups. The primary outcome was association of OS and PFS with temporalis CSA.

\section{Statistical methods}

Summary statistics for patient characteristics and segmentation metrics are presented as mean $\pm S D$ and frequency (percentage). Paired $t$ tests compared segmentation metrics obtained with different loss functions. To validate our model for the intended application of quantification of muscle as a measure of sarcopenia, similarity between CSA of manual and automated segmentations were evaluated using Pearson correlation.

We determined association of temporalis CSA and patient characteristics with Pearson correlation and independent $t$ tests. Kaplan-Meier survival analysis with log rank test was used to assess differences in OS and PFS by CSA group. The association between OS/PFS and CSA was tested in univariate Cox proportional hazard models and, where significance was found, in multivariate Cox models, controlling for age and sex (MGMT methylation status was not available for sufficient patients in the in-house data set so was not used as a covariate). Confidence intervals (Cls) for all estimates of risk were given as part of sensitivity analysis. Statistical significance was set at two-tailed $p$ value of $<0.05$. All analyses were performed using IBM SPSS v27 [43].

\section{RESULTS}

\section{Segmentation results}

Table 1 summarises quantitative performance metrics obtained by comparing muscle segmentations generated by the U-net model to manually segmented reference standard for alternative loss functions. The U-net trained with DL had highest performance, outperforming BCEL and HDL. BCEL achieved comparable performance to $\mathrm{DL}$, whereas performance differences between DL and HDL were significant across all metrics. Specifically, mean DSC was 0.912 for DL, significantly higher by $0.019(p<0.0005)$ and $0.119(p<0.0005)$ than BCEL and $\mathrm{HDL}$, respectively. $\mathrm{HD}$ achieved by $\mathrm{DL}$ was $1.81 \pm 0.39 \mathrm{~mm}$, similar to $1.83 \pm 0.34 \mathrm{~mm}$ $(p=0.79)$ for BCEL but a significant improvement over $2.18 \pm 0.44$ $\mathrm{mm}(p<0.0005)$ for HDL. Hence, for subsequent model training with a larger data set, we used a U-net model trained with DL.

The final U-net model trained on the full data set of $366 \mathrm{MRI}$ images from 132 patients segmented temporalis well. Figure 1 shows illustrative segmentation results.

Overall, the model achieved high accuracy in segmenting temporalis in the test set, with best mean DSC of $0.893 \pm 0.045, \mathrm{~J}$ of $0.809 \pm 0.072$ and HD of $1.889 \pm 0.354 \mathrm{~mm}$, indicating high overlap and close proximity between ground truth and automated segmentations. Mean precision and recall were similar- $0.867 \pm$ 0.077 and $0.926 \pm 0.046$ - suggesting no bias towards over- or under-segmentation.

There was strong correlation in muscle CSA between manual and automated segmentation $(r=0.902, p<0.0005)$. Average CSA error was $7.71 \pm 12.17 \%$, indicating comparable CSA measurement performance by the deep learning-based segmentation system relative to trained humans. A Bland-Altman plot (Fig. 2) shows no bias towards under- or over-segmentation.

Training on GPU took $10 \mathrm{~min}$ and the processing time for temporalis segmentation per novel case was $45 \mathrm{~ms}$, which was substantially faster than manual segmentation by a human rater, which required $10 \mathrm{~min}$ per case.

\section{Patient characteristics}

Ninety-six patients were included in the survival analysis: 45 from the in-house glioblastoma patient data set and 51 from the TCGAGBM data set. Subjects were aged $29-78$ and $23-76$ years, with median (interquartile range (IQR)) age of 55 (47-63) and 58 (51-66) in the in-house and TCGA-GBM data sets, respectively. There were 34 males (75.6\%) in the in-house data set and 33 males $(64.7 \%)$ in the TCGA-GBM data set. MGMT promoter was methylated in 18 (40.0\%) and unmethylated in 19 (42.2\%) patients in the in-house data set; 8 individuals had unknown methylation 


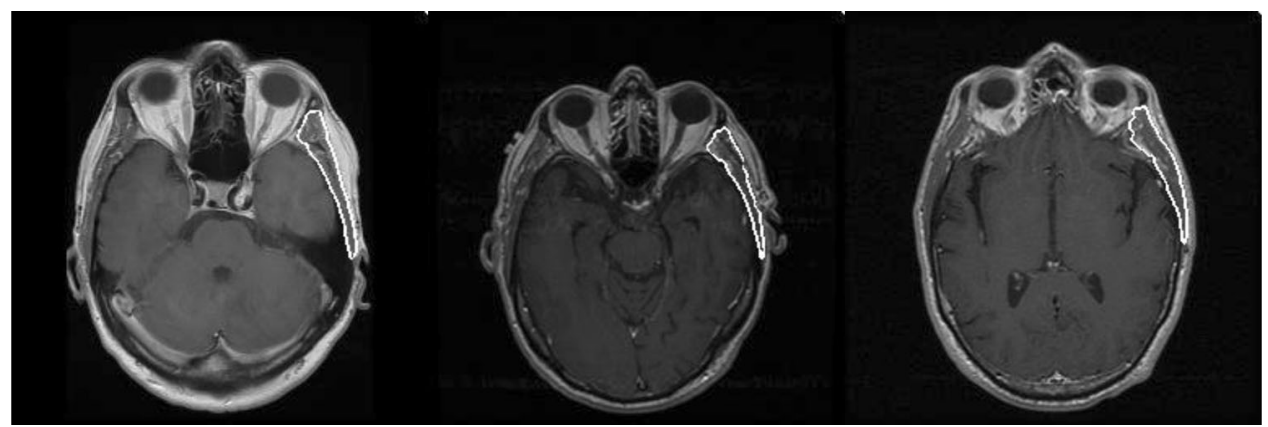

Fig. 1 Automated temporalis segmentations. Three representative test set MRI head images (T1 weighted + GAD contrast) with overlay of predicted temporalis muscle segmentations by the neural network.

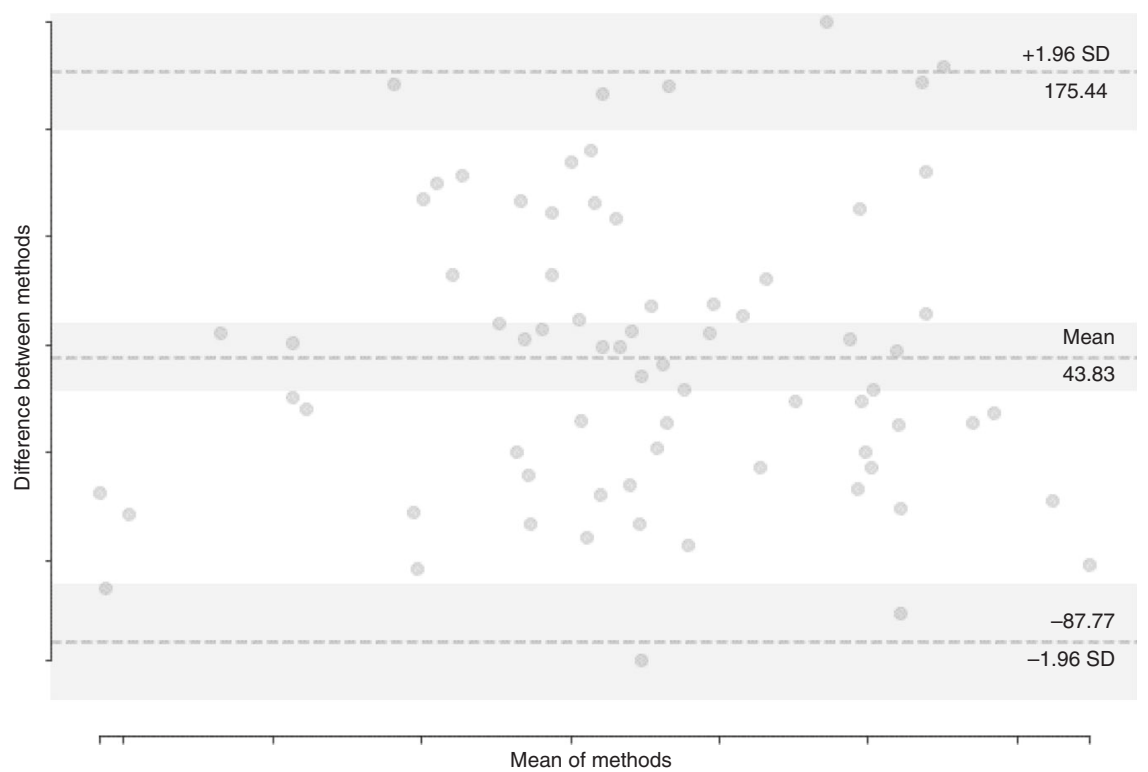

Fig. 2 Comparison of ground truth and automated temporalis segmentation muscle areas. Bland-Altman plot comparing cross-sectional areas of manual and predicted temporalis muscle segmentations in the test set.

status. Average follow-up was 19.2 months in the in-house data set and 15.2 months in the TCGA-GBM data set. Progression occurred in $42(93.3 \%)$ individuals and 40 (88.9\%) subjects had died at the time of analysis in the in-house data set, while 49 (96.1\%) patients died in the TCGA-GBM data set. Median (IQR) OS was 18.3 (12.0-24.8) months and PFS was 8.9 (5.9-15.4) months in the in-house data set. Median OS was 14.5 (6.9-20.1) months in the TCGA-GBM data set.

\section{CSA and patient characteristics}

Mean baseline temporalis CSA was $574 \pm 117 \mathrm{~mm}^{2}$ in the in-house glioblastoma patient data set and $605 \pm 137 \mathrm{~mm}^{2}$ in the TCGA-GBM data set. Both were normally distributed (Kolmogorov-Smirnov test $p>0.2$ for both). There was no significant relationship between temporalis CSA and age $(r=-0.213, p=0.16)$ in the in-house data set, but there was significant negative correlation between temporalis CSA and age $(r=-0.396, p=0.004)$ in the TGCA-GBM data set; Fig. 3 shows the distributions of CSA with age in the inhouse and TCGA-GBM data sets. Males had significantly higher CSA than females in both the in-house $\left(607 \pm 100\right.$ vs $472 \pm 109 \mathrm{~mm}^{2}, p<$ $0.0005)$ and TCGA-GBM data sets $\left(667 \pm 113\right.$ vs $492 \pm 101 \mathrm{~mm}^{2}, p<$ 0.0005).

\section{CSA and survival}

In the in-house glioblastoma patient data set, median baseline temporalis CSA was $588 \mathrm{~mm}^{2}$, which was used to dichotomise individuals to 'low' or 'high' CSA groups ( $n=22$ and $n=23$, respectively). OS and PFS was significantly longer in patients with high CSA (median OS 22.4 (16.9-27.4) vs 14.5 (9.7-21.1) months, $p$ $=0.011$; median PFS 14.3 (6.1-21.9) vs 6.4 (5.0-9.6) months, $p=$ 0.002) (Fig. 4a, b). In multivariate Cox models, adjusting for age and sex, CSA was an independently significant predictor for OS (hazard ratio (HR) $0.464,95 \% \mathrm{Cl} 0.218-0.988 ; p=0.046$ ) and PFS (HR 0.433, 95\% Cl 0.218-0.860; $p=0.017$ ) (Table 2). In the TCGAGBM data set, median baseline temporalis CSA was $595 \mathrm{~mm}^{2}$, with 26 and 25 patients in the 'low' and 'high' CSA groups, respectively. OS was significant longer in patients with high CSA (15.4 (8.9-22.8) vs 12.9 (4.1-15.6) months, $p=0.033$ ) (Fig. 4c). Multivariate Cox models for death for high CSA (vs low) yielded HR of $0.466(95 \% \mathrm{Cl}$ $0.235-0.925 ; p=0.029$ ) so CSA was independently significant for OS (Table 2). Age was also an independent prognostic factor in both data sets.

\section{DISCUSSION}

We have developed and validated a novel deep learning-based system for rapid and accurate temporalis muscle segmentation and quantification, with performance equivalent to but much quicker than trained humans. We show that temporalis muscle area is an independently significant prognostic marker for survival in GBM, corroborating previous evidence on TMW. To the best of our knowledge, this is the first study to demonstrate an 

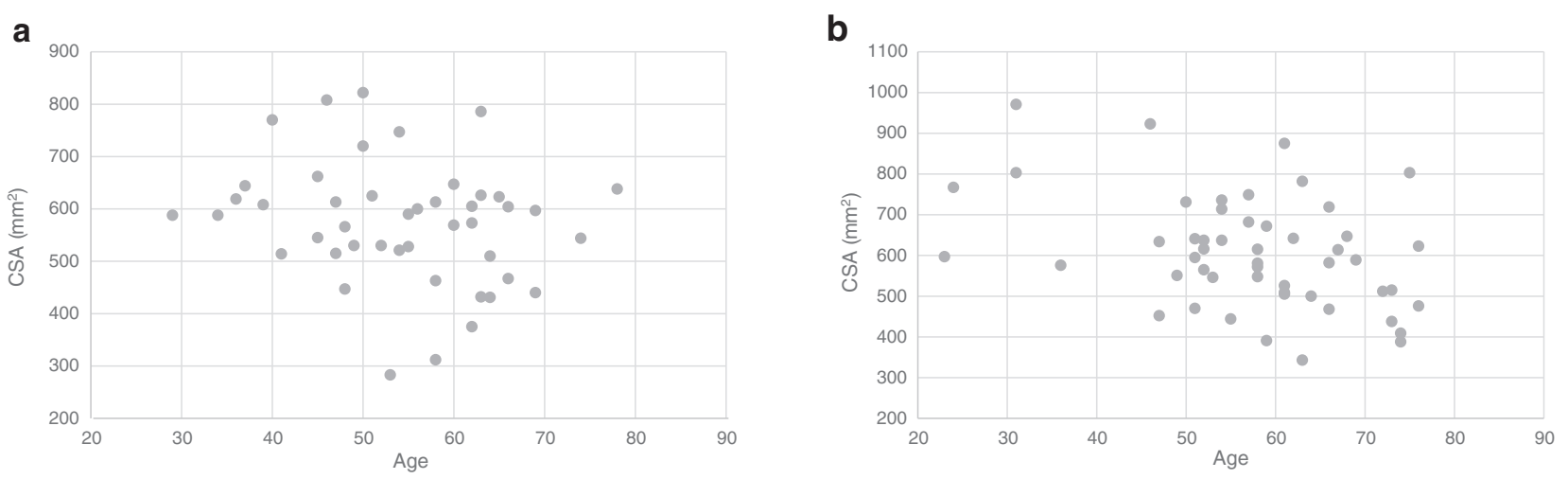

Fig. 3 Relationship between temporalis muscle area and age. Distribution of temporalis muscle area vs age in patients in the a in-house glioblastoma patient data set and b TCGA-GBM data set. CSA cross-sectional area.
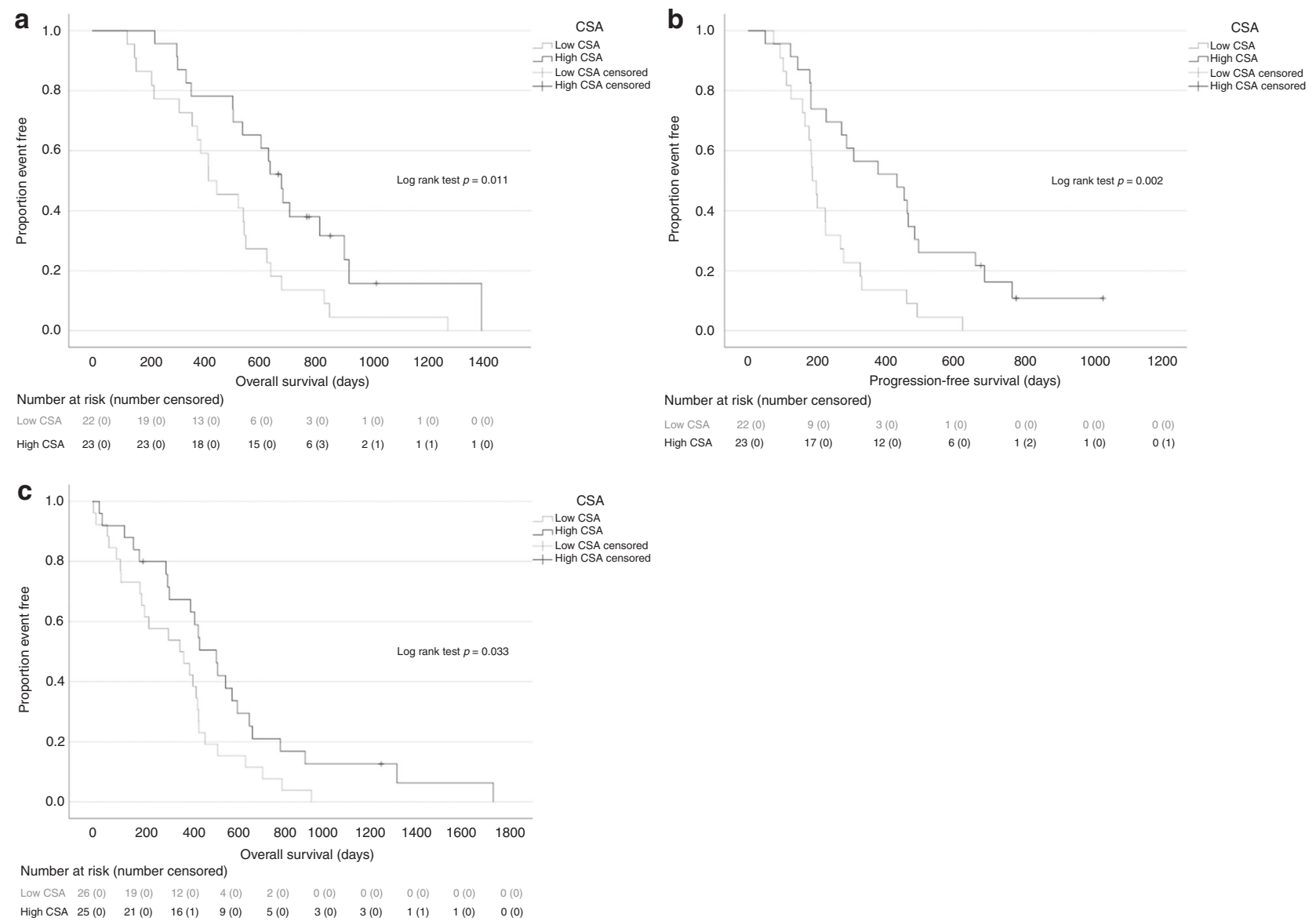

Fig. 4 Relationship between temporalis muscle area and survival in glioblastoma. Kaplan-Meier survival curves for overall survival (a) and progression-free survival (b) by temporalis muscle area group in the in-house glioblastoma patient data set and overall survival by temporalis muscle area group in the TCGA-GBM data set (c). CSA cross-sectional area.

association between a head/neck muscle-derived sarcopenia metric and clinical outcomes in cancer using deep learning. This is also one of the few studies, thus far, to combine both muscle segmentation and quantification using deep learning and show that such an automatically generated sarcopenia metric is significantly associated with oncological outcomes, supporting the possibility of a scalable approach to sarcopenia assessment on routine imaging in cancer.
Our technique produced generally better segmentation than previous approaches for the same muscle: previous models achieved DSC of 0.86 for masticatory muscles [44], 0.826 and 0.788 for masseter and temporalis [17] and 0.902 for temporalis [16], compared to DSC of 0.893 in this study. Our DSC is also comparable to previous models for segmentation of other muscles, for example, abdominal (DSC 0.90-0.97) [20-24], thigh (DSC 0.90-0.97) [18, 19] and shoulder (DSC 0.71-0.88) [26, 27]; our 
Table 2. Hazard ratios for overall and progression-free survival by temporalis muscle area group for in-house glioblastoma patient and TCGA-GBM data sets.

\begin{tabular}{|c|c|c|c|c|}
\hline \multirow[t]{2}{*}{ Patients } & \multirow{2}{*}{$\begin{array}{l}\text { Univariate } \\
\text { HR }(95 \% \mathrm{Cl})\end{array}$} & \multicolumn{3}{|c|}{ Multivariate } \\
\hline & & $p$ value & HR $(95 \% \mathrm{Cl})$ & $p$ value \\
\hline \multicolumn{5}{|c|}{ In-house glioblastoma patient data set-OS } \\
\hline Age & NA & NA & $1.036(1.002-1.070)$ & $0.036^{*}$ \\
\hline Sex & NA & NA & $1.430(0.622-3.287)$ & 0.399 \\
\hline \multicolumn{5}{|c|}{ In-house glioblastoma patient data set-PFS } \\
\hline Age & NA & NA & $1.028(0.998-1.059)$ & 0.067 \\
\hline Sex & NA & NA & $0.938(0.436-2.014)$ & 0.869 \\
\hline \multicolumn{5}{|c|}{ TCGA-GBM data set-OS } \\
\hline CSA & $0.536(0.299-0.961)$ & $0.036^{*}$ & $0.466(0.235-0.925)$ & $0.029^{*}$ \\
\hline Age & NA & NA & $1.029(1.004-1.056)$ & $0.025^{*}$ \\
\hline
\end{tabular}

All figures are $\mathrm{HR}(95 \% \mathrm{Cl})$.

OS overall survival, PFS progression-free survival, HR hazard ratio, CSA cross-sectional area, NA not applicable.

*Significant at $p<0.05$.

precision and recall of 0.867 and 0.926 is comparable to 0.93 and 0.91 for abdominal muscle [20]; and our HD of $1.889 \mathrm{~mm}$ is better than an existing model for masticatory muscles $(8.2 \mathrm{~mm})$ [44] as well as those for thigh $(2.3-8.2 \mathrm{~mm})$ [18] and lumbar abdominal (4.6-7.9 mm) muscles [22]. Thus, our model performed well, notwithstanding known challenges with facial muscles, e.g. homogeneous intensity to surrounding structures, shape complexity and significant anatomic variability. We found U-net trained using DL performed best, in keeping with DL being identified as superior for pelvic floor muscle segmentation [25].

There was a significant negative correlation between temporalis CSA and age in the TCGA-GBM data set but not in the in-house glioblastoma patient data set (although the trend was present so this difference between the two data sets may not be clinically significant). The former finding of a significant relationship is in line with general consensus of a negative correlation between muscle mass and age [45]. The latter finding of no significant relationship is similar to previous TMW studies [10-12, 14, 15]. It could be explained by disparity between chronological and biological age, the latter being more defined by frailty and physical condition. Thus, temporalis CSA may provide information not captured by age. We identified males to have significantly higher temporalis CSA than females, consistent with a study of lumbar abdominal muscle CSA [22], lending support to its use as a surrogate for skeletal muscle mass.

We found higher temporalis CSA was associated with significantly longer survival and time to progression in GBM with HR of 0.464 and 0.466 , and HR of 0.433 , consistent with studies of TMW reporting HRs of $0.41-0.79$ for OS and $0.46-0.77$ for PFS [1013]. Sarcopenia is a key feature of cancer-related cachexia. The mechanism linking lower temporalis CSA to worse survival outcomes likely reflects physical inactivity, nutritional deficiency and glioblastoma-related catabolic, paraneoplastic and inflammatory processes. Additionally, sarcopenic patients may tolerate surgery/chemoradiotherapy poorly, leading to toxicity or early discontinuation of therapy, and thus accelerated progression and death. Our results are in line with recently published studies this year, which show that muscle CSA at the L3 vertebral level on CT imaging, as assessed by a CNN, was significantly associated with survival in advanced cancer, and that greater muscle loss, as assessed by an Al-based volumetric technique, was a poor prognostic factor for OS $[46,47]$.

\section{Implications for clinical practice}

Our deep learning-based muscle segmentation and quantification tool has potential utility in bettering prognostic estimates in GBM and personalised treatment decisions, e.g. stratification to shorter, hypo-fractionated radiotherapy or temozolomide monotherapy, for which there is evidence of better outcomes in frail patients [48-50]. Our work suggests the possibility of using deep learningbased screening of sarcopenia in cancer care, without additional scanning time, cost or radiation exposure; this could inform muscle preservation interventions, such as nutrition, physiotherapy and pharmacotherapy [51-53]. Our tool is time and memory efficient, is applicable to large data sets and real-time assessment without specialist hardware and thus can feasibly be deployed in a routine clinical workflow.

\section{Study limitations}

This is a retrospective study and we had a limited data set. However, our training, validation and test data sets included MRI scans from four different data sets, three of which are external, and MR images at multiple orbital levels, heterogeneous in field of view and pixel resolution, acquired with machines from different manufacturers using different protocols; our model's robust performance indicates generalisability. In the survival analysis, we included patients from both our own institution and an external validation data set. However, given the limited number of patients, one can interpret the survival analysis as exploratory. The segmentation system was trained for $2 \mathrm{D}$ rather than $3 \mathrm{D}$ segmentation, common in muscle quantification due to the difficulty of creating good quality manual 3D segmentations, making 3D models more prone to error and reliant on postprocessing manual correction. 3D model training and application is also substantially slower with greatly increased computational memory cost, and it is unclear whether 3D temporalis segmentation would be of additional prognostic value. To ameliorate any potential loss of information with 2D segmentation (compared to 3D), we explored the relationship between temporalis muscle area at different orbital levels with survival, similar to previous studies that used the same anatomical landmarks [12]; the existence of a significant relationship at multiple orbital levels indicates generalisability. Our reason for exploring the robustness of the relationship at different orbital levels was also to facilitate our further work to develop a fully automated pipeline for temporalis 
segmentation with automatic slice selection based on orbital landmarks. However, we recognise that using different landmarks in the two data sets in survival analysis introduces a degree of inconsistency in our current methods and means we cannot directly combine the results of the two data sets; a future pipeline will automatically select slices based on a consistent landmark.

\section{CONCLUSION}

Our findings highlight temporalis muscle area as a non-invasive digital prognostic biomarker that can be automatically, rapidly and accurately assessed using deep learning, with feasible integration into routine clinical care. Our tool in its current form is semiautomated as it requires manual slice selection; however, we are currently developing a fully automated pipeline for temporalis segmentation including automatic slice selection using orbital landmarks. Future work will also involve prospective studies on larger cohorts.

\section{DATA AVAILABILITY}

The in-house data sets used and/or analysed during the current study are available from the corresponding author on reasonable request. The TCGA-GBM, IVY-GAP and REMBRANDT external data sets used and/or analysed during the current study are available from The Cancer Imaging Archive repository (https://wiki.cancerimagingarchive. net/display/Public/).

\section{CODE AVAILABILITY}

Code used in the study is available at our GitLab repository https://gitlab.com/ computational.oncology/temporalissegmentation and from the corresponding author on reasonable request.

\section{REFERENCES}

1. Brodbelt A, Greenberg D, Winters T, Williams M, Vernon S, Collins VP, et al. Glioblastoma in England: 2007-2011. Eur J Cancer. 2015;51:533-42.

2. Li J, Wang M, Won M, Shaw EG, Coughlin C, Curran WJ Jr, et al. Validation and simplification of the Radiation Therapy Oncology Group recursive partitioning analysis classification for glioblastoma. Int J Radiat Oncol Biol Phys. 2011;81:623-30.

3. Buentzel J, Heinz J, Bleckmann A, Bauer C, Rover C, Bohnenberger H, et al. Sarcopenia as prognostic factor in lung cancer patients: a systematic review and meta-analysis. Anticancer Res. 2019;39:4603-12.

4. Vergara-Fernandez O, Trejo-Avila M, Salgado-Nesme N. Sarcopenia in patients with colorectal cancer: a comprehensive review. World J Clin Cases. 2020;8:1188-202.

5. Zhang XM, Dou QL, Zeng Y, Yang Y, Cheng ASK, Zhang WW. Sarcopenia as a predictor of mortality in women with breast cancer: a meta-analysis and systematic review. BMC Cancer. 2020;20:172.

6. Rinninella E, Cintoni M, Raoul P, Pozzo C, Strippoli A, Bria E, et al. Muscle mass, assessed at diagnosis by L3-CT scan as a prognostic marker of clinical outcomes in patients with gastric cancer: a systematic review and meta-analysis. Clin Nutr. 2020;39:2045-54.

7. Allanson ER, Peng Y, Choi A, Hayes S, Janda M, Obermair A. A systematic review and meta-analysis of sarcopenia as a prognostic factor in gynecological malignancy. Int J Gynecol Cancer. 2020;30:1791-7. https://doi.org/10.1136/ijgc-2020001678.

8. Wong A, Zhu D, Kraus D, Tham T. Radiologically defined sarcopenia affects survival in head and neck cancer: a meta-analysis. Laryngoscope. 2020;131:333-41. https://doi.org/10.1002/lary.28616.

9. Leitner J, Pelster S, Schopf V, Berghoff AS, Woitek R, Asenbaum U, et al. High correlation of temporal muscle thickness with lumbar skeletal muscle cross-sectional area in patients with brain metastases. PLoS ONE. 2018;13: e0207849.

10. Cinkir HY, Er HC. Is temporal muscle thickness a survival predictor in newly diagnosed glioblastoma multiforme? Asia Pac J Clin Oncol. 2020. https://doi.org/ 10.1111/ajco.13369.

11. Furtner J, Genbrugge E, Gorlia T, Bendszus M, Nowosielski M, Golfinopoulos V, et al. Temporal muscle thickness is an independent prognostic marker in patients with progressive glioblastoma: translational imaging analysis of the EORTC 26101 trial. Neuro Oncol. 2019;21:1587-94.
12. Hsieh K, Hwang M, Estevez-Inoa G, Saraf A, Spina CS, Smith D, et al. Temporalis muscle width as a measure of sarcopenia correlates with overall survival in patients with newly diagnosed glioblastoma. J Radiat Oncol. 2019;8:379-87.

13. Zakaria H, Chandra A, Macki M, Robin A, Walbert T, Chang V, et al. INNV-21. In newly-diagnosed glioblastoma, frailty/sarcopenia predicts $30 \mathrm{~d}$ morbidity $\& 30 \mathrm{~d}$, $90 \mathrm{~d}$, and overall mortality as accurately as current standards. Neuro Oncol. 2019;21:vi135.

14. Furtner J, Berghoff AS, Schopf V, Reumann R, Pascher B, Woitek R, et al. Temporal muscle thickness is an independent prognostic marker in melanoma patients with newly diagnosed brain metastases. J Neurooncol. 2018;140:173-8.

15. Furtner J, Berghoff AS, Albtoush OM, Woitek R, Asenbaum U, Prayer D, et al. Survival prediction using temporal muscle thickness measurements on cranial magnetic resonance images in patients with newly diagnosed brain metastases. Eur Radiol. 2017;27:3167-73.

16. Ng HP, Hu QM, Ong SH, Foong KWC, Goh PS, Liu J, et al. Segmentation of the temporalis muscle from MR data. Int J Computer Assist Radiol Surg. 2017;2:19-30.

17. Rezaeitabar $Y$, Ulusoy I. Automatic 3D segmentation of individual facial muscles using unlabeled prior information. Int J Comput Assist Radiol Surg. 2012;7:35-41.

18. Kemnitz J, Baumgartner CF, Eckstein F, Chaudhari A, Ruhdorfer A, Wirth W, et al. Clinical evaluation of fully automated thigh muscle and adipose tissue segmentation using a U-Net deep learning architecture in context of osteoarthritic knee pain. MAGMA. 2020;33:483-93.

19. Yang YX, Chong MS, Tay L, Yew S, Yeo A, Tan CH. Automated assessment of thigh composition using machine learning for Dixon magnetic resonance images. MAGMA. 2016;29:723-31.

20. Edwards K, Chhabra A, Dormer J. Abdominal muscle segmentation from CT using a convolutional neural network. Proc SPIE Int Soc Opt Eng. 2020. https://doi.org/ 10.1117/12.2549406.

21. Park HJ, Shin Y, Park J, Kim H, Lee IS, Seo DW, et al. Development and validation of a deep learning system for segmentation of abdominal muscle and fat on computed tomography. Korean J Radiol. 2020;21:88-100.

22. Graffy PM, Liu J, Pickhardt PJ, Burns JE, Yao J, Summers RM. Deep learning-based muscle segmentation and quantification at abdominal $\mathrm{CT}$ : application to a longitudinal adult screening cohort for sarcopenia assessment. $\mathrm{Br} J$ Radiol. 2019;92:20190327.

23. Burns JE, Yao J, Chalhoub D, Chen JJ, Summers RM. A machine learning algorithm to estimate sarcopenia on abdominal CT. Acad Radiol. 2020;27:311-20.

24. Weston AD, Korfiatis P, Kline TL, Philbrick KA, Kostandy P, Sakinis T, et al. Automated abdominal segmentation of $C T$ scans for body composition analysis using deep learning. Radiology. 2019;290:669-79.

25. Feng F, Ashton-Miller JA, DeLancey JOL, Luo J. Convolutional neural networkbased pelvic floor structure segmentation using magnetic resonance imaging in pelvic organ prolapse. Med Phys. 2020. https://doi.org/10.1002/mp.14377.

26. Taghizadeh E, Truffer O, Becce F, Eminian S, Gidoin S, Terrier A, et al. Deep learning for the rapid automatic quantification and characterization of rotator cuff muscle degeneration from shoulder CT datasets. Eur Radiol. 2020. https://doi. org/10.1007/s00330-020-07070-7.

27. Conze PH, Brochard S, Burdin V, Sheehan FT, Pons C. Healthy versus pathological learning transferability in shoulder muscle MRI segmentation using deep convolutional encoder-decoders. Comput Med Imaging Graph. 2020;83:101733.

28. The Cancer Imaging Archive. The Cancer Imaging Archive (TCIA) Public Access. 2014. https://wiki.cancerimagingarchive.net/display/Public/. Accessed 9 Nov 2020.

29. Mauricaite R, Mi E, Chen J, Ho A, Pakzad-Shahabi L, Williams M. A fully automated deep learning pipeline to assess muscle mass in brain tumor patients. In: 2021 IEEE 34th international symposium on computer-based medical systems (CBMS). Piscataway, NJ: IEEE; 2021. p. 107-12.

30. Neurolmaging Tools \& Resources Collaboratory. MRIcron. 2019. https://www.nitrc. org/projects/mricron/. Accessed 9 Nov 2020.

31. Avants BB, Tustison NJ, Johnson HJ, Song G, Cook PA, Duda JT, et al. Advanced normalization tools in Python. 2017. https://github.com/ANTsX/ANTsPy. Accessed 20 Nov 2019.

32. Reinhold JC, Dewey BE, Carass A, Prince JL. Evaluating the impact of intensity normalization on $\{M R\}$ image synthesis. 2019. https://github.com/jcreinhold/ intensity-normalization. Accessed 20 Nov 2019.

33. ITK-SNAP. ITK-SNAP. 2008. http://www.itksnap.org/pmwiki/pmwiki.php. Accessed 20 Nov 2019.

34. Ronneberger $\mathrm{O}$, Fischer $\mathrm{P}$, Brox T. U-Net: convolutional networks for biomedical image segmentation. arXiv:1505.04597v1 [Preprint]. 2015 [cited 2015 May 18]. Available from: https://arxiv.org/abs/1505.04597.

35. GitHub. Implementation of deep learning framework - Unet, using Keras. 2018. https://github.com/zhixuhao/unet. Accessed 13 Jan 2020.

36. TensorFlow. https://www.tensorflow.org/. Accessed 13 Jan 2020.

37. Keras. https://keras.io/. Accessed 13 Jan 2020.

38. Python. https://www.python.org/. Accessed 5 Dec 2019. 
39. Yakubovskiy P. Segmentation models. 2019. https://github.com/qubvel/ segmentation_models. Accessed 20 Jan 2020.

40. Ribera J, Guera D, Chen Y, Delp EJ. Locating objects without bounding boxes arXiv:1806.07564v1 [Preprint]. 2018 [cited 2018 Jun 18]. Available from: https:// arxiv.org/abs/1806.07564.

41. GitHub. Weighted Hausdorff distance loss. 2019. https://github.com/ danielenricocahall/Keras-Weighted-Hausdorff-Distance-Loss. Accessed 20 Jan 2020.

42. OpenCV. https://opencv.org/. Accessed 17 Feb 2020

43. IBM. IBM SPSS software. https://www.ibm.com/uk-en/analytics/spss-statisticssoftware. Accessed 24 Jun 2020.

44. Chen W, Li Y, Dyer BA, Feng X, Rao S, Benedict SH, et al. Deep learning vs. atlasbased models for fast auto-segmentation of the masticatory muscles on head and neck CT images. Radiat Oncol. 2020;15:176.

45. Cruz-Jentoft AJ, Sayer AA. Sarcopenia. Lancet. 2019;393:2636-46.

46. van Seventer E, Marquardt JP, Troschel AS, Best TD, Horick N, Azoba C, et al. Associations of skeletal muscle with symptom burden and clinical outcomes in hospitalized patients with advanced cancer. J Natl Compr Cancer Netw. 2021. https://doi.org/10.6004/jnccn.2020.7618.

47. Kim SI, Yoon S, Kim TM, Cho JY, Chung HH, Song YS. Prognostic implications of body composition change during primary treatment in patients with ovarian cancer: a retrospective study using an artificial intelligence-based volumetric technique. Gynecol Oncol. 2021. https://doi.org/10.1016/j.ygyno.2021.05.004.

48. Roa W, Kepka L, Kumar N, Sinaika V, Matiello J, Lomidze D, et al. International atomic energy agency randomized phase III study of radiation therapy in elderly and/or frail patients with newly diagnosed glioblastoma multiforme. J Clin Oncol. 2015;33:4145-50.

49. Malmström A, Gronberg BH, Marosi C, Stupp R, Frappaz D, Schultz $H$, et al Temozolomide versus standard 6-week radiotherapy versus hypofractionated radiotherapy in patients older than 60 years with glioblastoma: the Nordic randomised, phase 3 trial. Lancet Oncol. 2012;13:916-26.

50. Chang EL, Yi W, Allen PK, Levin VA, Sawaya RE, Maor MH. Hypofractionated radiotherapy for elderly or younger low-performance status glioblastoma patients: outcome and prognostic factors. Int J Radiat Oncol Biol Phys. 2003;56:519-28.

51. Argilés JM, Busquets S, López-Soriano FJ, Costelli P, Penna F. Are there any benefits of exercise training in cancer cachexia? J Cachexia Sarcopenia Muscle. 2012;3:73-6.

52. Murphy RA, Mourtzakis M, Chu QSC, Baracos VE, Reiman T, Mazurak VC. Nutritional intervention with fish oil provides a benefit over standard of care for weight and skeletal muscle mass in patients with non-small cell lung cancer receiving chemotherapy. Cancer. 2011;117:1775-82.

53. Padhi D, Higano CS, Shore ND, Sieber P, Rasmussen E, Smith MR. Pharmacological inhibition of myostatin and changes in lean body mass and lower extremity muscle size in patients receiving androgen deprivation therapy for prostate cancer. J Clin Endocrinol Metab. 2014;99:E1967-75.

\section{ACKNOWLEDGEMENTS}

This work uses data provided by patients and collected by the NHS as part of their care and support. We are grateful to Dr Anastasia Gontsarova (Department of Radiotherapy, Charing Cross Hospital, Imperial College Healthcare NHS Trust) for providing training to $\mathrm{EM}$ and $\mathrm{RM}$ with regard to manual temporalis muscle segmentation. We are also grateful to Katherine Pike (Department of Radiotherapy, Charing Cross Hospital, Imperial College Healthcare NHS Trust) for providing EM and RM with access to the in-house glioblastoma MRI data set. A conference abstract of this work (Mi E, Mauricaite R, Pakzad-Shahabi L, Pike K, Williams M. Deep learningbased segmentation and quantification of temporalis muscle for sarcopenia assessment is an independent prognostic factor in glioblastoma) was presented at the National Cancer Research Institute Virtual Showcase 2020 on 2nd November 2020 and won the NCRI Award for best research. The methods of this study have in part been presented in a conference paper at the 2021 IEEE 34th International Symposium on Computer-Based Medical Systems. It is reproduced, with permission, from Mauricaite, R., Mi, E., Chen, J., Ho, A., Pakzad-Shahabi, L., Williams, M. A fully automated deep learning pipeline to assess muscle mass in brain tumour patients. 2021 IEEE 34th International Symposium on Computer-Based Medical Systems (CBMS), pp. 107-112 (2021). https://doi.org/10.1109/CBMS52027.2021.00095. @ 2021 IEEE.

\section{AUTHOR CONTRIBUTIONS}

Substantial contributions to the conception or design of the work or the acquisition, analysis or interpretation of data for the work; drafting the manuscript and revising it critically for important intellectual content; approval of the final submitted version; agreement to be accountable for all aspects of the work and ensuring questions related to the accuracy or integrity of any part of the work are appropriately investigated, resolved and the resolution documented in the literature: EM, RM, LP-S, $\mathrm{JC}, \mathrm{AH}, \mathrm{MW}$. EM designed the study, supervised the manual segmentation of MR images, conducted training, optimisation and testing of the neural network for segmentation, conducted the CSA and survival data analyses and interpreted the data. RM contributed to study design, extracted, preprocessed and did manual segmentation of MR images, conducted training, optimisation, testing of the neural network for segmentation and contributed to the CSA and survival data analyses and interpretation. LP-S collected the survival data. JC supervised manual segmentation of $\mathrm{MR}$ images. $\mathrm{AH}$ collected the MR image data. MW conceived the study and supervised data collection, analysis and interpretation, had full access to all the data in the study and had final responsibility for the decision to submit for publication.

\section{FUNDING}

MW receives funding from the National Institute for Health Research Imperial Biomedical Research Centre and Imperial Cancer Research UK Centre; LP-S receives funding from Brain Tumour Research and the Brain Tumour Research Campaign. The authors received no specific funding for this work. No funding source had a role in study design or conduct, data collection, analysis or interpretation, manuscript writing, review or approval or decision to submit it for publication.

\section{COMPETING INTERESTS}

The authors declare no competing interests.

\section{ETHICS APPROVAL AND CONSENT TO PARTICIPATE}

The study had REC/HRA approval by London - Bloomsbury Research Ethics Committee (reference: 19/LO/1763 IRAS ID: 265404) and was conducted in accordance with this committee's regulations and the Declaration of Helsinki.

\section{ADDITIONAL INFORMATION}

Supplementary information The online version contains supplementary material available at https://doi.org/10.1038/s41416-021-01590-9.

Correspondence and requests for materials should be addressed to Matt Williams.

Reprints and permission information is available at http://www.nature.com/ reprints

Publisher's note Springer Nature remains neutral with regard to jurisdictional claims in published maps and institutional affiliations.

\begin{abstract}
Open Access This article is licensed under a Creative Commons

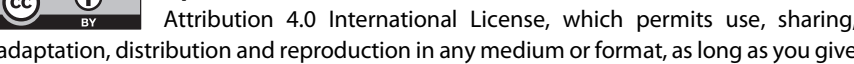
appropriate credit to the original author(s) and the source, provide a link to the Creative Commons license, and indicate if changes were made. The images or other third party material in this article are included in the article's Creative Commons license, unless indicated otherwise in a credit line to the material. If material is not included in the article's Creative Commons license and your intended use is not permitted by statutory regulation or exceeds the permitted use, you will need to obtain permission directly from the copyright holder. To view a copy of this license, visit http://creativecommons. org/licenses/by/4.0/.
\end{abstract}

(c) The Author(s) 2021 